\title{
Patients with chronic mesenteric ischemia have an altered sublingual microcirculation
}

\author{
This article was published in the following Dove Press journal: \\ Clinical and Experimental Gastroenterology
}

\author{
Jihan Harki ${ }^{1, *}$ \\ Mustafa Suker ${ }^{2, *}$ \\ M Sherezade Tovar-Doncel ${ }^{3}$ \\ Louisa JD van Dijk' \\ Désirée van Noord' \\ Casper $\mathrm{HJ}$ van Eijck ${ }^{2}$ \\ Marco J Bruno' \\ Ernst J Kuipers ${ }^{1,4}$ \\ Can Ince ${ }^{5}$ \\ 'Department of Gastroenterology and \\ Hepatology, ${ }^{2}$ Department of Surgery, \\ Erasmus MC University Medical \\ Center Rotterdam, Rotterdam, \\ the Netherlands; ${ }^{3}$ Department of \\ Anesthesiology, University Hospital \\ Rio Hortega, Valladolid, Spain; \\ ${ }^{4}$ Department of Internal Medicine, \\ ${ }^{5}$ Department of Intensive Care, \\ Erasmus MC University Medical \\ Center, Rotterdam, the Netherlands \\ *These authors contributed equally to \\ this work
}

\begin{abstract}
Background: Little is known about the microcirculatory alterations in patients with chronic mesenteric ischemia (CMI). We hypothesized that patients with CMI have an impaired microcirculatory function and show an oral microcirculatory response after caloric challenge compared to healthy controls.
\end{abstract}

Methods: All patients and controls received the standard workup for CMI. Sublingual microcirculation was evaluated before (T0) and 20 minutes after (T1) feeding. The total vessel density (TVD; $\mathrm{mm} / \mathrm{mm}^{2}$ ), perfused vessel density (PVD; $\mathrm{mm} / \mathrm{mm}^{2}$ ), proportion of perfused vessels (PPV; \%) and microvascular flow index (MFI; AU) were assessed.

Results: We included 12 patients (63.2 years [IQR 48.8-70.4 years], 67\% males) and 12 controls (32.7 years [IQR 27.7-38.1 years], 42\% males). At baseline, patients with CMI had a decreased PPV of the sublingual small vessels (median $84.8 \%$ vs $95.7 \%, P=0.006$ ), PPV of all vessels (PPV median $85.4 \%$ vs $95.3 \%, P=0.007$ ) and microvascular flow index of all vessels (MFIa; median 3.00 vs $2.80, P=0.039$ ) compared to healthy controls. After caloric challenge, PVD increased significantly in both small vessels (perfused vessel density of the small vessels [PVDs]) and all vessels (perfused vessel density of all vessels [PVDa]; PVDs [T0]) median 16.3 [IQR 13.3-22.1] vs [T1] median 19.9 [IQR 14.2-26.2], $P=0.008$; PVDa [T0] median 19.1 [IQR 16.2-23.6] vs [T1] median 22.2 [IQR 16.5-28.9], $P=0.02$; proportion of perfused vessels of the small vessels (PPVs; [T0] median 84.8\% [IQR 75.3-90.4] vs [T1] median 91.0\% [IQR 80.1-93.8], $P=0.010$ ). In contrast, no significant changes in microcirculatory parameters were observed after caloric challenge in healthy controls.

Conclusion: Patients with CMI have an impaired sublingual microcirculation at baseline and show a significant response in the sublingual microcirculation after caloric challenge, whereas healthy controls have a normal microcirculation at baseline and show no reactive response upon a caloric challenge as seen in CMI patients. Sublingual microcirculation visualization may offer a rapid noninvasive method to identify patients at risk for having CMI.

Keywords: chronic mesenteric ischemia, ischemia, sublingual microcirculation, Cytocam-IDF, diagnosis

\section{Introduction}

Chronic mesenteric ischemia (CMI) results from insufficient blood supply to the stomach, small intestine and colon, leading to decreased mucosal perfusion and oxygenation. ${ }^{1}$ In most cases, CMI is caused by stenosis of the supplying arteries due to atherosclerosis. Chronic heart failure and pulmonary conditions can further contribute to or predominate CMI (so-called nonocclusive mesenteric ischemia $[\mathrm{NOMI}]) .^{2}$
Correspondence: Jihan Harki Department of Gastroenterology and Hepatology, Erasmus MC University Medical Center Rotterdam, PO Box 2040, Rotterdam, 3015 CE, the Netherlands

$\mathrm{Tel}+3 \mathrm{I} \quad 107040704$

Fax +31 107035429

Email j.harki@erasmusmc.nl 
Patients with CMI have insufficient postprandial increase in the splanchnic blood flow after ingesting food,which causes a misbalance between oxygen delivery and metabolic demand and often results in postprandial discomfort. ${ }^{3}$ Until recently, it was thought that CMI could only occur in the presence of at least two occluded mesenteric arteries, but studies have shown that a significant stenosis of a single artery can also lead to clinically relevant gastrointestinal ischemia. ${ }^{4,5}$ In patients with single-vessel CMI, the symptoms are thought to be related to insufficient collateral circulation. ${ }^{2}$ Another underlying cause might be that patients with CMI have microcirculatory alterations associated with systemic disorders of the circulatory system. The majority of patients with CMI have hypertension, diabetes and other cardiovascular conditions. ${ }^{6}$ Therefore, microvascular pathology in this patient group is very likely, which might explain why single-vessel stenosis can lead to clinical symptoms even in the presence of seemingly sufficient collateral circulation.

However, the microcirculation has not been studied in patients with CMI. In patients with hemodynamic shock and acute gastrointestinal ischemia, a correlation between intestinal ischemia and sublingual microcirculatory alterations has been observed during investigations of buccal, sublingual and intestinal mucosa. ${ }^{7-10}$ Another study reported decreased microvessel density and erythrocyte velocity in both the sublingual and gut regions. ${ }^{11}$ Sublingual capillary perfusion correlated with the hypoxic state of the gastric mucosa in these patients. ${ }^{9,12,13}$ The sublingual $\mathrm{PCO}_{2}$ in patients with septic shock paralleled gastric $\mathrm{PCO}_{2}$ as measured using gastric tonometry. ${ }^{14}$ Similarly, in elective stoma surgery complicated by sepsis, sublingual and ileal microcirculatory alterations were correlated.

To our knowledge, no study has investigated the relationship between sublingual microcirculation and hemodynamic changes in patients with CMI. Based on the findings in patients with acute ischemia, we formulated the hypothesis that 1) patients show an oral microcirculatory response after caloric challenge different from that of healthy individuals and 2) patients with CMI have impaired microcirculatory function compared with that of healthy individuals.

\section{Methods}

\section{Study population}

Patients with confirmed CMI were enrolled between September 2014 and August 2015. The current established approach for diagnosing CMI is based on three main components. The first component includes assessment of medical history, clinical symptoms and physical examination. The second component concerns radiological imaging of the mesenteric arteries, and the third component aims at detection of mucosal ischemia by means of a functional test. All patients and procedures are discussed by a dedicated multidisciplinary team consisting of a vascular surgeon, intervention radiologist and gastroenterologist, all specialized in CMI, leading to a final expert-based consensus diagnosis.

The diagnosis of CMI is established if a patient fulfills two of the three following criteria: ${ }^{15}$

1. Distinctive clinical presentation including presence of postprandial pain and otherwise unexplained weight loss of $>5 \%$ of the normal body weight.

2. Significant stenosis of $>70 \%$ of at least one of the mesenteric arteries demonstrated by radiological evaluation.

3. Mucosal ischemia detected by tonometry or visible light spectroscopy (VLS). Mucosal ischemia was defined as mucosal oxygen saturation $<58 \%,<62 \%$, or $<63 \%$ for measurements in the duodenum, duodenal bulb and antrum of the stomach, respectively, based on previous assessments in patients and healthy controls. .,16,17 $^{2}$

A definitive diagnosis of CMI is made after persistent relief of symptoms on follow-up after treatment, which included revascularization in patients with stenotic CMI and vasodilatory medication in patients with NOMI.

This is not the ideal gold standard; however, it is currently the most reliable way to establish CMI. For single-vessel disease, all three criteria should apply.,16,17

Nonsmoking healthy subjects with patent gastrointestinal arteries as determined by abdominal duplex ultrasound, no gastrointestinal complaints and unremarkable medical history were asked to participate in our study on a voluntary basis.

All patients provided written informed consent prior to participation to the study. Approval for the study was obtained from the institutional review board of the Erasmus MC University Medical Center Rotterdam, the Netherlands (MEC number 2013-003).

\section{Assessment of microcirculation}

We measured sublingual microcirculation according to the latest consensus guidelines using the Cytocam-IDF device before (T0) and 20 minutes after (T1) enteral feeding. ${ }^{18}$ Patients and healthy controls received enteral feeding 
through an endoscopically placed feeding tube in the antrum. Through the feeding tube, patients were given 300 $\mathrm{mL}$ of compound liquid food (Nutrison Energy ${ }^{\circledR}$; Nutricia Advanced Medical Nutrition, Zoetermeer, the Netherlands), a solution of carbohydrates, protein and fat with a low volume and a high caloric content ( $450 \mathrm{kcal})$. At each time point, microcirculation was assessed at three different sublingual mucosal locations for a minimum of 4 seconds per sequence as previously described. ${ }^{19}$ The locations concerned the sublingual mucosal tissue surface at the floor of the mouth adjacent to the lingual frenulum. Great care was taken to clear saliva before image acquisition. Film sequences were repeatedly taken until three sequences of good quality were obtained. The quality of the images was assessed using the Massey score for microcirculation image quality. ${ }^{20}$ Videoimage analysis was performed blindly by one independent researcher (MSTD) according to protocol using Automated Vascular Analysis (AVA) 3.0. ${ }^{21}$ The following parameters were obtained for small vessels and all vessels: total vessel density (TVD; $\mathrm{mm} / \mathrm{mm}^{2}$ ), perfused vessel density (PVD; $\mathrm{mm} / \mathrm{mm}^{2}$ ), proportion of perfused vessels (PPV; \%) and microvascular flow index (MFI; AU). The definition for PVD is the total length $(\mathrm{mm})$ of perfused vessels divided by the total mucosal surface, and PPV is defined as the proportion of perfused vessels divided by the total vessels on the recorded surface.

\section{Statistical analyses}

Baseline characteristics were calculated using descriptive statistics. Data were expressed as median and IQR or count and percentage (\%), as appropriate. A Wilcoxon signed-rank test was performed to study the differences between baseline and postprandial parameters of the microcirculation in CMI patients and healthy controls. Statistical analysis was performed using SPSS 21.0 program (SPSS Inc., Chicago, IL, USA). A two-sided $P$-value of $<0.050$ was considered as significant.

\section{Results}

\section{Patient characteristics}

During the study period, 49 consecutive patients with clinical suspicion of CMI were eligible for the study. Informed consent was received from 31 (63\%) patients. Reasons for nonparticipation were mostly poor condition of the patient or anxiety regarding undergoing multiple tests. Three $(6 \%)$ patients failed to complete this workup, leaving a total of $28(57 \%)$ participants in the study. The results of the diagnostic workup of these 28 patients were discussed by a multidisciplinary expert panel. A consensus diagnosis of CMI was made in $14(50 \%)$ patients, whereas CGI was ruled out in $14(50 \%)$ patients.

During follow-up (median 3.0 [IQR 1-6] months), two patients were lost to follow-up, leaving a total of 12 patients with CMI (11 patients with occlusive CMI and one patient with NOMI). For the characteristics of patients and controls, see Table 1 .

Table I Baseline characteristics of CMI patients and controls

\begin{tabular}{|c|c|c|}
\hline & $\operatorname{CMI}(n=12)$ & Controls $(n=12)$ \\
\hline Age $^{a}$ & $63.2(48.8-70.4)$ & $32.7(27.7-38.1)$ \\
\hline \multicolumn{3}{|l|}{ Gender } \\
\hline Male & $8(67)$ & $5(42)$ \\
\hline Female & $4(33)$ & $7(58)$ \\
\hline BMI $\left(\mathbf{k g} / \mathrm{m}^{2}\right)^{\mathrm{a}}$ & $24.2(21.5-28.3)$ & $22.3(20.4-24.8)$ \\
\hline \multicolumn{3}{|l|}{ Symptomatology } \\
\hline Abdominal pain & $10(83)$ & 0 \\
\hline Postprandial pain & $9(75)$ & 0 \\
\hline Exercise-induced pain & $4(33)$ & 0 \\
\hline Weight loss & $7(58)$ & 0 \\
\hline Weight loss (kg/months) ${ }^{\mathrm{a}}$ & $2.0(I . I-3.0)$ & 0 \\
\hline Diarrhea & $2(17)$ & 0 \\
\hline Nausea & $5(42)$ & 0 \\
\hline Duration of symptoms (months) & $10.0(6.0-21.0)$ & 0 \\
\hline \multicolumn{3}{|l|}{ Cardiovascular comorbidity } \\
\hline History of CVD & $8(67)$ & 0 \\
\hline CAD alone & $0(0)$ & 0 \\
\hline PAD alone & $4(33)$ & 0 \\
\hline Both & $4(33)$ & 0 \\
\hline Hypertension & $7(58)$ & 0 \\
\hline DM type II & $3(25)$ & 0 \\
\hline Hypercholesterolemia & $6(50)$ & 0 \\
\hline Family history of CVD & $5(42)$ & 0 \\
\hline Smoking & $10(83)$ & $4(40)$ \\
\hline Current & $6(50)$ & $I(8)$ \\
\hline Former & $4(33)$ & $3(25)$ \\
\hline Pack years ${ }^{\mathrm{a}}$ & $162(0-356)$ & 0 \\
\hline Alcohol use & $6(50)$ & $8(67)$ \\
\hline Alcohol (U/day) ${ }^{c}$ & $0(0-1.8)$ & $0.6(0-1.0)$ \\
\hline \multicolumn{3}{|l|}{ Mesenteric artery stenosis ${ }^{d}$} \\
\hline No stenosis & I (8) & 0 \\
\hline Single artery stenosis & $4(33)$ & 0 \\
\hline Multi artery stenosis & $7(58)$ & 0 \\
\hline \multicolumn{3}{|l|}{ VLS measurement } \\
\hline Antrum ${ }^{\mathrm{a}}$ & $58.5(53.5-59.0)$ & $61.5(60.0-63.0)$ \\
\hline Duodenal bulb & $53.0(48.8-54.0)$ & $59.0(54.0-63.8)$ \\
\hline Descending duodenum ${ }^{\mathrm{a}}$ & $49.5(45.3-52.8)$ & $51.5(47.5-56.0)$ \\
\hline
\end{tabular}

Notes: Values represent the number and percentage of patients unless otherwise specified. aMedian, IQR. 'Includes current and former smokers. Median, range. 'Stenosis of the mesenteric arteries is defined as $>70 \%$ lumen reduction on CTA or MRA.

Abbreviations: BMI, body max index; CAD, cardial arterial disease; CMI, chronic mesenteric ischemia; CTA, computed tomography angiography; CVD, cardiovascular disease; DM, diabetes mellitus; MRA, magnetic resonance angiography; PAD, peripheral arterial disease; VLS, visible light spectroscopy. 


\section{Sublingual microcirculation}

A snapshot of a recorded image of the sublingual microcirculation of a healthy volunteer obtained during the study is shown in Figure 1. Absolute data and ranges for the microvascular variables of all patients and controls are provided in Table 2. Compared to the baseline (T0), perfused vessel density of the small vessels (PVDs) and perfused vessel density of all vessels (PVDa) significantly increased in patients with CMI after caloric challenge (T1; PVDs median 16.3 $\mathrm{mm} / \mathrm{mm}^{2}$ [IQR $13.3-22.1 \mathrm{~mm} / \mathrm{mm}^{2}$ ] vs $19.9 \mathrm{~mm} / \mathrm{mm}^{2}$ [IQR 14.2-26.2 mm/ $\left.\mathrm{mm}^{2}\right], P=0.008$; PVDa median $19.1 \mathrm{~mm} / \mathrm{mm}^{2}$ [IQR $16.2-23.6 \mathrm{~mm} / \mathrm{mm}^{2}$ ] vs $22.2 \mathrm{~mm} / \mathrm{mm}^{2}$ [IQR $16.5-28.9$ $\left.\mathrm{mm} / \mathrm{mm}^{2}\right], P=0.020$; Table 3 and Figure 2). Furthermore, there was a significant increase in the proportion of perfused vessels of the small vessels (PPVs) between baseline (T0) and after caloric challenge (T1; median 84.8\% [IQR 75.3\%-90.4\%] vs 91.0\% [IQR 80.1\%-93.8\%], $P=0.010$ ). This did not pertain to the proportion of perfused vessels of all vessels (PPVa; median 85.4\% [IQR 79.8\%-91.5\%] vs 90.4\% [IQR 81.4-94.4], $P=0.050$; Figure 3 ). In addition, there was a nonsignificant increase in total vessel density of the small vessels (TVDs) after caloric challenge (median $20.8 \mathrm{~mm} / \mathrm{mm}^{2}$ [IQR 17.6-23.8 $\mathrm{mm} / \mathrm{mm}^{2}$ ] vs $22.4 \mathrm{~mm} / \mathrm{mm}^{2}$ [IQR $17.8-32.2 \mathrm{~mm} / \mathrm{mm}^{2}$ ], $P=0.090$ ). In contrast, there was no significant difference in microcirculation between the values at baseline and after caloric challenge in healthy volunteers (Figure 4 and Table 3 ).

At baseline (T0), patients with CMI had a decreased PPVs (median $84.8 \%$ vs $95.7 \%, P=0.006$ ), PPVa (median $85.4 \%$ vs $95.3 \%, P=0.007$; Figure 4 ) and microvascular flow index of all vessels (MFIa; median 2.8 vs 3.0, $P=0.039$ ) compared with those of healthy controls. After caloric challenge (T1), patients with CGI had a decreased PPVa compared

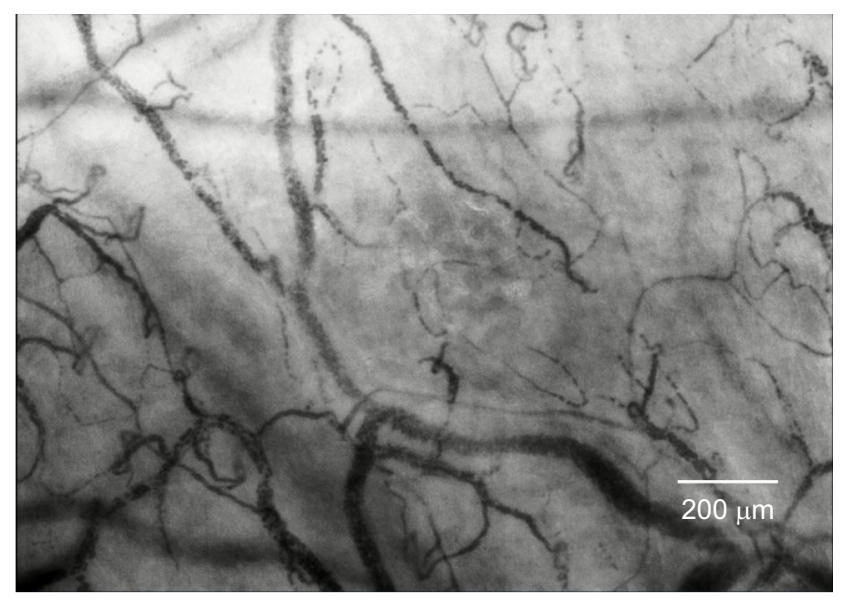

Figure I Recorded image of the sublingual microcirculation of a healthy volunteer obtained during the study. to the healthy controls (median $90.4 \%$ vs $94.8 \%, P=0.039$ ) and MFIa (median 2.9 vs 3.0, $P=0.014$ ). The difference in sublingual microcirculatory parameters between patients and healthy controls is shown in Table 3. Video clips of an example of sublingual microcirculation of a healthy individual and of CMI patients can be found in Videos S1-S4.

\section{Discussion}

The main findings of our study were that: 1) at baseline, patients with CMI have a significantly lower PPV and a lower microcirculatory flow index than those of healthy controls; 2) increased metabolic demand induced by food intake caused in CMI patients an increase in PVD and in PPV, whereas this did not occur in the healthy controls; and 3) despite the increase in PVD and PPV of the CMI patients, levels did not reach the normal levels of the healthy controls. These latter findings are consistent with the idea that the compromised microcirculation of CMI patients requires recruitment of the microcirculation to meet the metabolic demand imposed by food intake, but that this does not occur in health where there is sufficient microcirculatory reserve to meet such a demand.

Previous studies in patients with CMI focused on macrovascular alterations. ${ }^{22-24}$ Patients with CMI have an impaired postprandial increase in the splanchnic blood flow, leading to insufficient oxygen delivery to meet the metabolic demand of the gastrointestinal tract. ${ }^{3}$ Another underlying cause might be that patients with CMI have microcirculatory alterations associated with systemic disorders of the circulatory system, which may also be attributed to the presence of clinical symptoms. ${ }^{6}$ Systemic microvascular pathology in this patient group is very likely, given the high association with cardiovascular conditions. This might explain why single-vessel stenosis can lead to clinical symptoms even in the presence of sufficient collateral circulation. We therefore hypothesized that patients with CMI may also exhibit microcirculatory alterations relative to healthy controls. We also hypothesized that sublingual microcirculation is responsive to enteral caloric challenges and that, based on preexisting microcirculatory alterations in CMI, microcirculation provides a way to indirectly assess intestinal microcirculation.

Our study shows that patients with CMI experience a significant need to recruit the microcirculation to meet the metabolic stress induced by the caloric challenge and thus have a response to the ingestion of food of highly caloric food. However, this hyperemic response to the ingestion of food was not seen in the healthy volunteers. Therefore, it seems that patients with CMI have a poor microcirculation to begin with, despite the increase in microcirculatory parameters after 
Table 2 Individual data of sublingual microcirculation of patients with CMI and healthy controls

\begin{tabular}{|c|c|c|c|c|c|c|c|c|c|}
\hline \multirow[t]{2}{*}{ Patient } & \multirow{2}{*}{$\begin{array}{l}\text { Time } \\
\text { point }^{\text {a }}\end{array}$} & \multicolumn{8}{|l|}{ Parameters } \\
\hline & & $\begin{array}{l}\text { TVDs } \\
\left(\mathrm{mm} / \mathrm{mm}^{2}\right)\end{array}$ & $\begin{array}{l}\text { TVDa } \\
\left(\mathrm{mm} / \mathrm{mm}^{2}\right)\end{array}$ & $\begin{array}{l}\text { PVDs } \\
\left(\mathrm{mm} / \mathrm{mm}^{2}\right)\end{array}$ & $\begin{array}{l}\text { PVDa } \\
\left(\mathrm{mm} / \mathrm{mm}^{2}\right)\end{array}$ & $\begin{array}{l}\text { PPVs } \\
\text { (\%) }\end{array}$ & $\begin{array}{l}\text { PPVa } \\
\text { (\%) }\end{array}$ & $\begin{array}{l}\text { MFIs } \\
\text { (AU) }\end{array}$ & $\begin{array}{l}\text { MFla } \\
\text { (AU) }\end{array}$ \\
\hline \multirow[t]{2}{*}{ I } & 0 & 19.13 & 24.22 & 17.19 & 22.25 & 90.50 & 92.27 & 2.83 & 2.94 \\
\hline & I & 18.76 & 21.81 & 16.29 & 19.24 & 86.48 & 87.66 & 3.00 & 2.84 \\
\hline \multirow[t]{2}{*}{2} & 0 & 15.36 & 18.00 & 13.58 & 16.17 & 87.58 & 89.20 & 3.00 & 2.61 \\
\hline & I & 13.87 & 15.01 & 13.32 & 14.46 & 95.97 & 96.17 & 3.00 & 2.90 \\
\hline \multirow[t]{2}{*}{3} & 0 & 17.64 & 21.08 & 13.27 & 16.71 & 75.34 & 79.35 & 2.67 & 2.56 \\
\hline & I & $17.5 \mid$ & 21.93 & 14.33 & 17.95 & 82.80 & 83.28 & 2.50 & 2.80 \\
\hline \multirow[t]{2}{*}{4} & 0 & 23.95 & 25.18 & 21.78 & 23.01 & 91.44 & 91.78 & 3.00 & 2.97 \\
\hline & 1 & 34.90 & 38.03 & 32.31 & 35.44 & 92.92 & 93.46 & 3.00 & 3.00 \\
\hline \multirow[t]{2}{*}{5} & 0 & 23.45 & 25.04 & 22.25 & 23.84 & 94.80 & 95.20 & 3.00 & 3.00 \\
\hline & I & 25.42 & 28.44 & 24.96 & 27.98 & 98.05 & 98.36 & 3.00 & 3.00 \\
\hline \multirow[t]{2}{*}{6} & 0 & 20.54 & 24.13 & 10.82 & 13.43 & 52.52 & 55.79 & 1.58 & 1.94 \\
\hline & I & 19.90 & 23.94 & 12.58 & 16.21 & 62.63 & 66.41 & 2.33 & 2.58 \\
\hline \multirow[t]{2}{*}{7} & 0 & 21.11 & 23.87 & 15.35 & 17.00 & 73.64 & 72.98 & 2.75 & 2.50 \\
\hline & I & 16.50 & $|8.3|$ & 15.47 & 17.26 & 92.81 & 93.74 & 3.00 & 2.94 \\
\hline \multirow[t]{2}{*}{8} & 0 & 31.68 & 34.26 & 28.80 & 31.37 & 89.98 & 90.70 & 3.00 & 3.00 \\
\hline & 1 & 34.45 & 35.76 & 31.09 & 32.20 & 90.40 & 90.08 & 3.00 & 2.93 \\
\hline \multirow[t]{2}{*}{9} & 0 & 11.88 & 15.65 & 8.98 & 12.75 & 75.25 & 81.11 & 2.83 & 2.70 \\
\hline & I & 18.52 & 20.77 & $|4.2|$ & 16.10 & 77.20 & 78.16 & 2.89 & 2.49 \\
\hline \multirow[t]{2}{*}{10} & 0 & 23.66 & 25.68 & 19.24 & 21.25 & 81.95 & 83.09 & 2.67 & 2.89 \\
\hline & I & 27.78 & 27.78 & 25.43 & 25.24 & 91.57 & 90.79 & 3.00 & 2.89 \\
\hline \multirow[t]{2}{*}{ II } & 0 & 28.70 & 31.06 & 22.72 & 25.08 & 79.47 & 81.06 & 2.75 & 2.75 \\
\hline & I & 33.67 & 36.33 & 26.51 & 29.17 & 79.22 & 80.79 & 2.58 & 2.82 \\
\hline \multirow[t]{2}{*}{12} & 0 & 17.56 & 19.01 & 15.12 & 16.26 & 88.39 & 87.66 & 3.00 & 2.85 \\
\hline & I & 24.85 & 27.32 & 23.42 & 25.88 & 94.11 & 94.62 & 3.00 & 3.00 \\
\hline \multicolumn{10}{|l|}{ Control } \\
\hline \multirow[t]{2}{*}{ I } & 0 & 33.52 & 34.51 & 32.70 & 33.68 & 97.39 & 97.46 & 3 & 3 \\
\hline & I & 27.14 & 28.13 & 25.32 & 26.30 & 92.41 & 92.68 & 3 & 3 \\
\hline \multirow[t]{2}{*}{2} & 0 & 23.11 & 25.21 & 20.52 & $22.6 I$ & 86.80 & 88.16 & 3 & 2.75 \\
\hline & I & 20.27 & 22.52 & 19.47 & 21.72 & 95.39 & 96.05 & 3 & 3 \\
\hline \multirow[t]{2}{*}{3} & 0 & 24.46 & 25.55 & 23.49 & 24.58 & 95.29 & 94.34 & 3 & 3 \\
\hline & 1 & 29.36 & 31.45 & 28.23 & 30.32 & 96.30 & 96.51 & 3 & 3 \\
\hline 4 & 0 & 16.38 & 16.88 & 16.34 & 16.84 & 99.77 & 99.78 & 3 & 3 \\
\hline & I & 17.01 & 17.74 & 15.49 & 16.22 & 91.21 & 91.94 & 2.83 & 2.78 \\
\hline 5 & 0 & 20.58 & 22.81 & 13.00 & 14.58 & 61.76 & 62.43 & 2.75 & 2.47 \\
\hline & I & 19.70 & 23.01 & 16.92 & 20.24 & 82.52 & 84.60 & 2.75 & 2.86 \\
\hline 6 & 0 & 16.33 & 19.05 & 13.88 & 17.66 & 89.69 & 91.99 & 2.83 & 2.74 \\
\hline & I & 24.56 & 26.22 & 24.56 & 26.38 & 100.00 & 100.00 & 3 & 3 \\
\hline 7 & 0 & 20.94 & 22.54 & 19.04 & 20.35 & 90.96 & 90.21 & 3 & 3 \\
\hline & I & 23.87 & 24.20 & 22.14 & 22.47 & 92.68 & 92.78 & 3 & 3.03 \\
\hline 8 & 0 & 19.63 & 20.32 & 19.54 & 20.23 & 99.60 & 99.60 & 3 & 3 \\
\hline & I & 16.85 & 19.73 & 16.59 & 19.28 & 98.18 & 97.66 & 3.13 & 3 \\
\hline 9 & 0 & 24.50 & 26.35 & 22.19 & 24.04 & 90.81 & 91.21 & 3 & 3 \\
\hline & 1 & 23.32 & 23.32 & 19.85 & 23.09 & 98.91 & 99.03 & 3 & 3 \\
\hline 10 & 0 & 23.36 & 25.88 & 23.28 & 25.81 & 99.68 & 99.70 & 3 & 3 \\
\hline & I & 19.56 & 22.78 & 18.28 & 21.41 & 92.66 & 93.57 & 3 & 3 \\
\hline 11 & 0 & 25.15 & 25.88 & 24.14 & 24.88 & 96.11 & 96.20 & 3 & 2.93 \\
\hline & I & 26.13 & 29.46 & 24.34 & 27.67 & 90.78 & 92.32 & 3 & 3 \\
\hline 12 & 0 & 23.48 & 25.33 & 23.06 & 24.87 & 97.79 & 97.73 & 3 & 3 \\
\hline & I & 23.26 & 25.90 & 23.14 & 25.59 & 99.46 & 98.83 & 3 & 3 \\
\hline
\end{tabular}

Notes: ${ }^{a}$ Time point 0 is the baseline before caloric challenge, and time point $\mathrm{I}$ is after caloric challenge. ${ }^{\mathrm{b}} \mathrm{P}<0.050$ is considered as significant.

Abbreviations: CMI, chronic mesenteric ischemia; MFla, microvascular flow index of all vessels; MFls, microvascular flow index of the small vessels; PPVa, proportion of perfused vessels of all vessels; PPVs, proportion of perfused vessels of the small vessels; PVDa, perfused vessel density of all vessels; PVDs, perfused vessel density of the small vessels; TVDa, total vessel density of all vessels; TVDs, total vessel density of the small vessels. 
Table 3 Difference in sublingual microcirculatory parameters before and after caloric challenge

\begin{tabular}{|c|c|c|c|c|c|}
\hline \multirow[t]{2}{*}{ Parameters } & \multirow[t]{2}{*}{ Time point ${ }^{a}$} & \multicolumn{2}{|l|}{ Patients $(n=12)$} & \multicolumn{2}{|c|}{ Controls $(n=12)$} \\
\hline & & Median (IQR) & $P$-value ${ }^{b}$ & Median (IQR) & $P$-value ${ }^{b}$ \\
\hline \multirow[t]{2}{*}{ TVDs $\left(\mathrm{mm} / \mathrm{mm}^{2}\right)$} & 0 & $20.8(17.6-23.8)$ & 0.090 & $23.2(20.1-24.5)$ & 0.810 \\
\hline & I & $22.4(17.8-32.2)$ & & $23.3(19.6-25.3)$ & \\
\hline \multirow[t]{2}{*}{ TVDa $\left(\mathrm{mm} / \mathrm{mm}^{2}\right)$} & 0 & $24.2(19.5-25.6)$ & 0.180 & $25.3(21.4-25.9)$ & 0.750 \\
\hline & I & $25.6(21.0-33.9)$ & & $23.8(22.7-27.2)$ & \\
\hline \multirow[t]{2}{*}{ PVDs $\left(\mathrm{mm} / \mathrm{mm}^{2}\right)$} & 0 & $16.3(13.3-22.1)$ & 0.008 & $21.4(17.7-23.4)$ & 1.00 \\
\hline & I & $19.9(14.2-26.2)$ & & $21.0(17.6-24.5)$ & \\
\hline \multirow[t]{2}{*}{ PVDa $\left(\mathrm{mm} / \mathrm{mm}^{2}\right)$} & 0 & $19.1(16.2-23.6)$ & 0.020 & $23.3(18.9-24.9)$ & 0.580 \\
\hline & 1 & $22.2(16.5-28.9)$ & & $22.8(20.8-26.3)$ & \\
\hline \multirow[t]{2}{*}{ PPVs (\%) } & 0 & $84.8(75.3-90.4)$ & 0.010 & 95.7 (90.3-98.7) & 0.430 \\
\hline & I & $91.0(80.1-93.8)$ & & $94.0(91.8-98.5)$ & \\
\hline \multirow[t]{2}{*}{ PPVa (\%) } & 0 & 85.4 (79.8-91.5) & 0.050 & 95.3 (90.7-98.7) & 0.430 \\
\hline & I & $90.4(81.4-94.4)$ & & $94.8(92.5-98.2)$ & \\
\hline \multirow[t]{2}{*}{ MFls (AU) } & 0 & $2.8(2.7-3.0)$ & 0.170 & $3.0(3.0-3.0)$ & 0.790 \\
\hline & I & $3.0(2.7-3.0)$ & & $3.0(3.0-3.0)$ & \\
\hline \multirow[t]{2}{*}{ MFla (AU) } & 0 & $2.8(2.6-3.0)$ & 0.140 & $3.0(2.8-3.0)$ & 0.120 \\
\hline & I & $2.9(2.8-3.0)$ & & $3.0(3.0-3.0)$ & \\
\hline
\end{tabular}

Notes: ${ }^{\mathrm{a} T i m e}$ point 0 is at baseline before caloric challenge, and time point $\mathrm{I}$ is after caloric challenge. ${ }^{\mathrm{b}} \mathrm{P}<0.050$ is considered as significant.

Abbreviations: MFla, microvascular flow index of all vessels; MFls, microvascular flow index of the small vessels; PPVa, proportion of perfused vessels of all vessels; PPVs, proportion of perfused vessels of the small vessels; PVDa, perfused vessel density of all vessels; PVDs, perfused vessel density of the small vessels; TVDa, total vessel density of all vessels; TVDs, total vessel density of the small vessels.

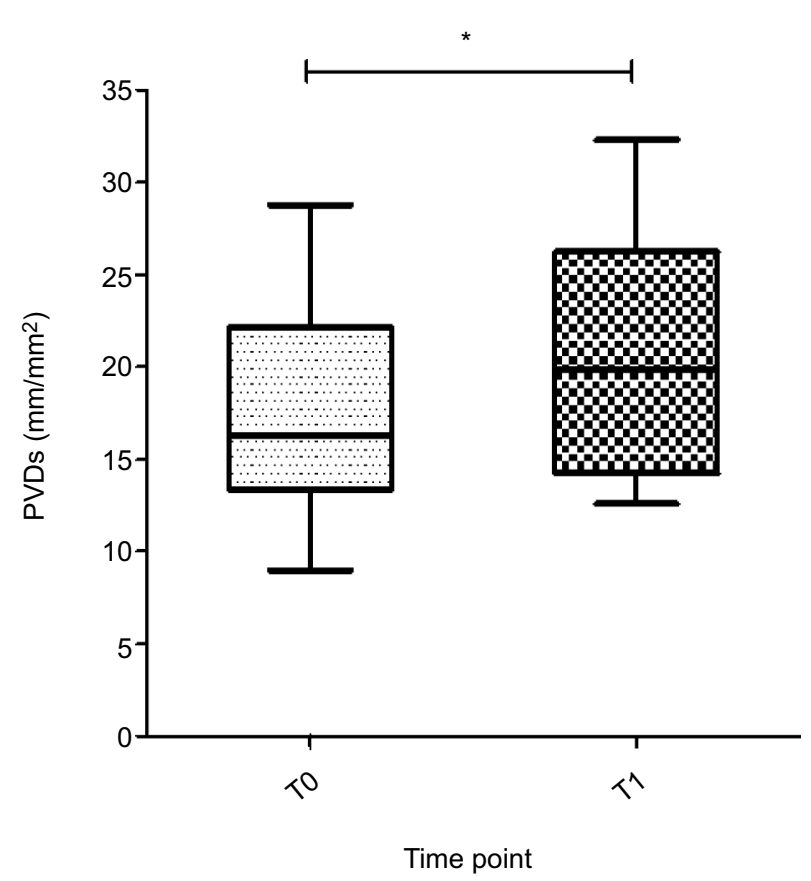

Figure 2 PVDs $\left(\mathrm{mm} / \mathrm{mm}^{2}\right)$ of all patients with CMI at baseline (T0) and after caloric challenge (TI).

Note: Boxes represent medians with IQRs, whiskers extend to the most and least extreme scores and $*$ denotes $<0.050$.

Abbreviations: CMI, chronic mesenteric ischemia; PVDs, perfused vessel density of the small vessels.

a caloric challenge to compensate for the microcirculatory shortcoming. In contrast, it seems that healthy controls begin with normal microcirculation; therefore, no compensatory microcirculatory mechanism is initiated to ingest food. From this reasoning, it follows that microcirculation before

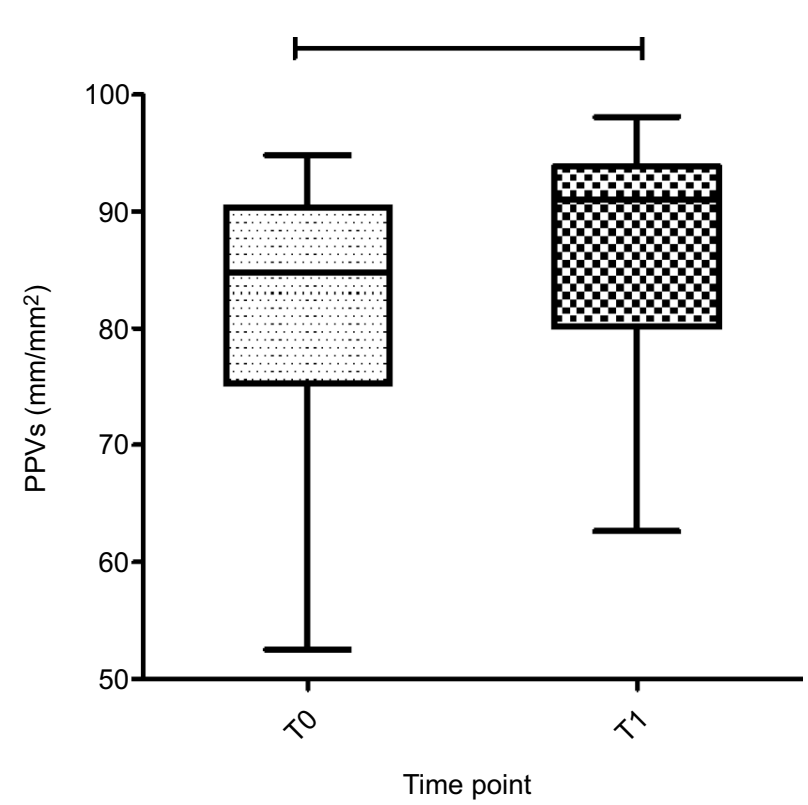

Figure 3 PPVs $\left(\mathrm{mm} / \mathrm{mm}^{2}\right)$ of all patients with CMI at baseline (T0) and after caloric challenge (TI).

Note: Boxes represent medians with IQR, whiskers extent to the most and least extreme scores and $*$ denotes $<0.050$.

Abbreviations: CMI, chronic mesenteric ischemia; PPVs, proportion of perfused vessels of the small vessels.

food exposure in CMI patients is lower than that in healthy individuals and that the compensatory increase after food challenge does not reach normal levels. Further studies including more patients and healthy controls using the current protocol are needed to further investigate this matter. 


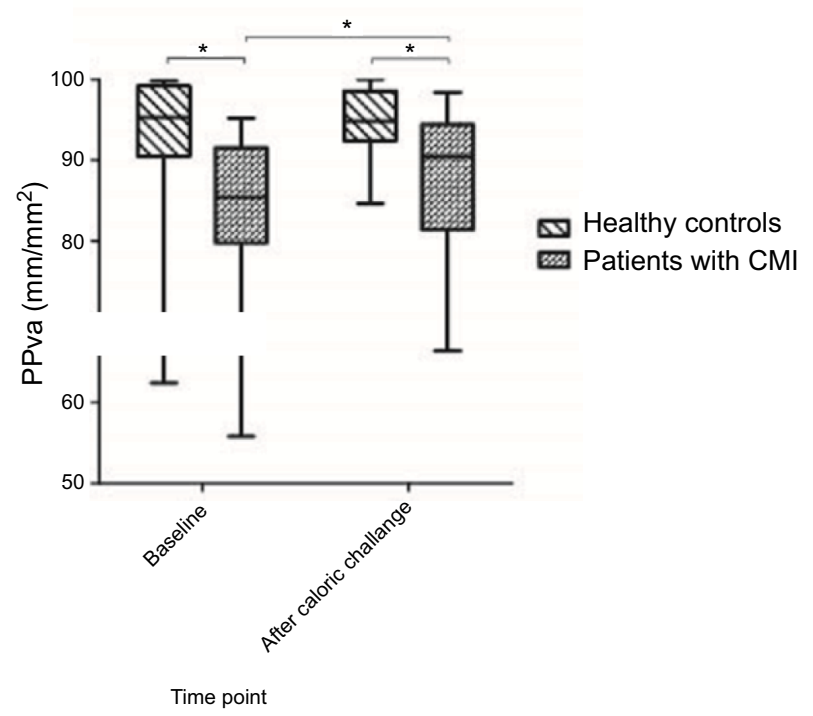

Figure $4 \mathrm{PPVs}\left(\mathrm{mm} / \mathrm{mm}^{2}\right)$ of patients with $\mathrm{CMI}$ and healthy controls at baseline (T0) and after caloric challenge (TI).

Note: Boxes represent medians with IQR, whiskers extend to the most and least extreme scores and $*$ denotes $<0.050$.

Abbreviations: CMI, chronic mesenteric ischemia; PPVs, proportion of perfused vessels of the small vessels.

Our study has several limitations. The first limitation is the diagnosis of CMI. The diagnosis of CMI is a clinical challenge. This is first because patients with CMI may present with a variety of symptoms. Furthermore, there is no single, accurate test to diagnose or exclude CMI. The role of clinical symptoms alone in the diagnosis of CMI is limited. $^{22,25}$ The combination of postprandial pain, weight loss and presence of an abdominal bruit is known as the classical triad of CGI. Indeed, our study could have benefitted from a careful assessment of clinical symptoms related to CMI following our challenge with a meal and measurement of the microcirculatory response, and this omission could be considered as a limitation of our study. This is however only present in $16 \%-21 \%$ of patients and has limited predictive value for diagnosing CGI. ${ }^{22,26,27}$ This supports the current diagnostic approach that combines assessment of clinical symptoms with radiological imaging of the gastrointestinal arteries and functional testing by tonometry or VLS. ${ }^{2}$ This is not the ideal gold standard; however, it is currently the most reliable way to establish CMI. Patients with CMI and the healthy controls were under conscious sedation with intravenous midazolam and fentanyl at the time of the second sublingual microcirculatory measurements after caloric challenge but not during the baseline sublingual measurements. A recent study showed that the sublingual microcirculation in patients with shock was greater after the infusion of midazolam. ${ }^{28}$ In this study, the PPV was significantly greater after the infusion of midazolam (PPV median 96.4\% [IQR 93.7-97.6] vs 92.7\% [IQR 88.3-94.7], $P<0.005)$. Therefore, one could hypothesize that the hyperemic response in our patients after caloric challenge might be partly due to the effect of midazolam. This phenomenon was not observed in the healthy controls. Therefore, the effect of conscious sedation seems limited. Furthermore, the small sample size of patients and controls could be affected by confounders. The major four confounders in this study are cardiovascular comorbidities, age, smoking and diabetes. It is suggested that a possible confounder in the results could be the difference in age between the control group (mean age 32 years) and CMI patients (mean age 63 years). However, a recent study by Kanoore Edul et $\mathrm{al}^{29}$ showed that that there is no relation between age and sublingual microvascular vascular density. In addition, as one-third of the patients have generalized arterial atherosclerosis, this could affect the sublingual microcirculation. However, to find patients with CMI without any history of CVD would however be very difficult and would decrease the sample size in our study. Yet in our study, one-third of our patients had no cardiovascular morbidity at all. Additionally, half of the patients are current smokers, which could induce microcirculatory changes in the mouth as suggested by Morozumi et al. ${ }^{30}$ However, there are a number of critical comments that can be made about this study. The study concerns the effects of smoking cessation on flow (eg, most likely simply related to the amount of circulating carbon monoxide). The first is that a similar study had been carried out using the same technique and location and it was found the opposite in that there was no difference in gingival microcirculatory flow between smokers and nonsmokers. ${ }^{31}$ It should also be pointed out that the laser Doppler technique used in these studies has known short comings in assessing the microcirculation. This includes that it measures an unspecific physiological compartment and is a nonquantitative measurement expressed in arbitrary units. It does not measure the microcirculatory vessel density in a quantitative manner $\left(\mathrm{eg}, \mathrm{mm} / \mathrm{mm}^{2}\right)$ as measured by handheld video microscopy where there is direct observation of the microcirculation. We investigated the effect of smoking using handheld video microscopy in a previous study in the same location as the study by Morozumi et al (the gingiva), and in contrast to their study, we found no effects of smoking on the gingival microcirculation. ${ }^{32}$ Another study looking at the sublingual microcirculation using handheld video microscopy confirmed these findings where no effect was found on sublingual microcirculation. ${ }^{29}$ This latter study also showed that smoking and age do not affect the microcirculation. ${ }^{29}$ However, it did show microcirculatory changes 
in patients with hypertension compared to patients without hypertension. However, these changes were only seen for TVD. If we compare the PPVs in the CMI patients with this study, there is a difference of $85.4 \%$ vs $100 \%$. We cannot compare the other microcirculatory parameters as we used the IDF device and the study of Kanoore et al used the stream dark field device. Indeed, diabetes mellitus (DM) could have affected the microcirculation; however, only $25 \%$ of the CMI patients had DM type 2, and therefore, we do think the influence is minimal on the microcirculation in our study. All these confounders can affect the microcirculatory differences between the patients and healthy controls; however, this does not affect the microcirculatory differences within a patient. Future research should focus on better matching of patients and controls by including controls known with DM and a smoking history to set the correct baseline from which a comparison can be established, thereby eliminating these confounders. A different type of confounder could have been the physiological metabolic response to a meal in the maxillomandibular musculature in the form of a reactive hypereamic response following ingestion of food. Indeed, a delayed reactive hyperemic response has been measured in the masseter in volunteers following clenching. ${ }^{33}$ However, this hyperemic response was found to be over within 2 minutes, whereas our microcirculatory measurements were performed 20 minutes after the administration of the meal, so we do not think this response could be a confounder. In addition, we administered the meal not orally but instead by a feeding tube placed in the antrum of the stomach. Finally, this study focuses more on the physiology of the microcirculation before and after a meal in CMI patients; therefore, no definite conclusions should be drawn from the differences with the healthy controls as no other studies have been performed on microcirculation after a meal. More and larger studies are needed to confirm the findings of our study.

To our knowledge, this is the first study to investigate the sublingual microcirculation in patients with CMI. Patients with CMI have less perfused capillaries in the sublingual microcirculation before food exposure compared with that of healthy controls and that the compensatory increase after food challenge in these patients does not reach normal levels. Furthermore, we found patients with CMI experience a significant increase in PVD and PPV after stimulation with food and therefore have a hyperemic response to the ingestion of food. However, this response is not sufficient to increase the microcirculation of CMI patients to the microcirculatory state of healthy volunteers with or without caloric challenge.
This study provides us additional insight into the relationship between the microcirculation and hemodynamic changes in the gastrointestinal tract. These findings may lead to the development of diagnostic methodology to identify patients at risk of having CMI. To establish such a clinical screening methodology for the identification of patients at risk for CMI, larger studies focusing on the sensitivity and specificity of measured alterations in the sublingual microcirculation would have to be undertaken.

\section{Abbreviations list}

CAD, cardial arterial disease

CMI, chronic mesenteric ischemia

CTA, computed tomography angiography

CVD, cardiovascular disease

$\mathrm{DM}$, diabetes mellitus

NOMI, nonocclusive mesenteric ischemia

MFIa, microvascular flow index of all vessels

MFIs, microvascular flow index of small vessels

MRA, magnetic resonance angiography

PAD, peripheral arterial disease

PPVa, proportion of perfused vessels of all vessels

PPVs, proportion of perfused vessels of the small vessels

PVDa, perfused vessel density of all vessels

PVDs, perfused vessel density of the small vessels

TVDa, total vessel density of all vessels

TVDs, total vessel density of the small vessels

VLS, visible light spectroscopy

\section{Acknowledgments}

The abstract of this paper was presented as a poster at the United European Gastroenterology Week 2017; October 30, 2017; Barcelona, Spain; and as a poster with interim findings at the Digestive Disease Week 2016; May 21, 2016; San Diego, CA, USA. The poster's abstract has been published. ${ }^{34}$

\section{Disclosure}

Professor Ince has developed stream dark field imaging, the predecessor of the device used in this study, and is listed as an inventor on related patents commercialized by MicroVision Medical (MVM) under a license from the Academic Medical Center in Amsterdam, the Netherlands. He has been a consultant for MVM in the past but has not been involved with this company for $>5$ years now and holds no shares. Braedius Medical, a company owned by a relative of Professor Ince, has developed and designed a handheld microscope called CytoCam-IDF imaging used in this study. Professor Ince has no financial relation with Braedius Medical of any sort, ie, 
never owned shares, or received consultancy or speaker fees from Braedius Medical. The authors report no other conflicts of interest in this work.

\section{References}

1. Brandt LJ, Feuerstadt P, Longstreth GF, Boley SJ, American College of Gastroenterology. ACG clinical guideline: epidemiology, risk factors, patterns of presentation, diagnosis, and management of colon ischemia (CI). Am J Gastroenterol. 2015;110(1):18-44.

2. Mensink PB, Moons LM, Kuipers EJ. Chronic gastrointestinal ischaemia: shifting paradigms. Gut. 2011;60(5):722-737.

3. Mitchell EL, Moneta GL. Mesenteric duplex scanning. Perspect Vasc Surg Endovasc Ther. 2006;18(2):175-183.

4. Mensink PB, van Petersen AS, Kolkman JJ, Otte JA, Huisman AB, Geelkerken RH. Gastric exercise tonometry: the key investigation in patients with suspected celiac artery compression syndrome. $J$ Vasc Surg. 2006;44(2):277-281.

5. van Noord D, Kuipers EJ, Mensink PB. Single vessel abdominal arterial disease. Best Pract Res Clin Gastroenterol. 2009;23(1):49-60.

6. Sana A, van Noord D, Mensink PB, et al. Patients with chronic gastrointestinal ischemia have a higher cardiovascular disease risk and mortality. Atherosclerosis. 2012;224(1):235-241.

7. Chierego M. Verdant C and De Backer D. Microcirculatory alterations in critically ill patients. Minerva Anestesiol. 2006;72(4):199-205.

8. Marik PE. Sublingual capnography: a clinical validation study. Chest. 2001;120(3):923-927.

9. Verdant CL, De Backer D, Bruhn A, et al. Evaluation of sublingual and gut mucosal microcirculation in sepsis: a quantitative analysis. Crit Care Med. 2009;37(11):2875-2881.

10. Pellis T, Weil MH, Tang W, Sun S, Csapozi P, Castillo C. Increases in both buccal and sublingual partial pressure of carbon dioxide reflect decreases of tissue blood flows in a porcine model during hemorrhagic shock. J Trauma. 2005;58(4):817-824.

11. Dubin A, Pozo MO, Ferrara G, et al. Systemic and microcirculatory responses to progressive hemorrhage. Intensive Care Med. 2009;35(3):556-564.

12. Edul VS, Ince C, Navarro N, et al. Dissociation between sublingual and gut microcirculation in the response to a fluid challenge in postoperative patients with abdominal sepsis. Ann Intensive Care. 2014;4:39.

13. Creteur J, de Backer D, Sakr Y, Koch M, Vincent JL. Sublingual capnometry tracks microcirculatory changes in septic patients. Intensive Care Med. 2006;32(4):516-523.

14. Povoas HP, Weil MH, Tang W, Sun S, Kamohara T, Bisera J. Decreases in mesenteric blood flow associated with increases in sublingual $\mathrm{PCO} 2$ during hemorrhagic shock. Shock. 2001;15(5):398-402.

15. Kolkman JJ, Geelkerken RH. Diagnosis and treatment of chronic mesenteric ischemia: An update. Best Pract Res Clin Gastroenterol. 2017;31(1):49-57.

16. van Noord D, Sana A, Moons LM, et al. Combining radiological imaging and gastrointestinal tonometry: a minimal invasive and useful approach for the workup of chronic gastrointestinal ischemia. Eur J Gastroenterol Hepatol. 2013;25(6):719-725.

17. van Noord D, Sana A, Benaron DA, et al. Endoscopic visible light spectroscopy: a new, minimally invasive technique to diagnose chronic GI ischemia. Gastrointest Endosc. 2011;73(2):291-298.
18. Ince $\mathrm{C}$, Boerma EC, Cecconi M, et al. Second consensus on the assessment of sublingual microcirculation in critically ill patients: results from a task force of the European Society of Intensive Care Medicine. Intensive Care Med. 2018;44(3):281-299.

19. de Backer D, Hollenberg S, Boerma C, et al. How to evaluate the microcirculation: report of a round table conference. Crit Care. 2007;11(5):R101.

20. Massey MJ, Larochelle E, Najarro G, et al. The microcirculation image quality score: development and preliminary evaluation of a proposed approach to grading quality of image acquisition for bedside videomicroscopy. J Crit Care. 2013;28(6):913-917.

21. Dobbe JG, Streekstra GJ, Atasever B, van Zijderveld R, Ince C. Measurement of functional microcirculatory geometry and velocity distributions using automated image analysis. Med Biol Eng Comput. 2008;46(7):659-670.

22. Sana A, Vergouwe Y, van Noord D, et al. Radiological imaging and gastrointestinal tonometry add value in diagnosis of chronic gastrointestinal ischemia. Clin Gastroenterol Hepatol. 2011;9(3):234-241.

23. Harki J, Vergouwe Y, Spoor JA, et al. Diagnostic Accuracy of the Combination of Clinical Symptoms and CT or MR Angiography in Patients With Chronic Gastrointestinal Ischemia. J Clin Gastroenterol. 2017;51:e39-e47.

24. Moawad J, Gewertz BL. Chronic mesenteric ischemia. Clinical presentation and diagnosis. Surg Clin North Am. 1997;77(2):357-369.

25. Ter Steege RW, Sloterdijk HS, Geelkerken RH, Huisman AB, van der Palen J, Kolkman JJ. Splanchnic artery stenosis and abdominal complaints: clinical history is of limited value in detection of gastrointestinal ischemia. World J Surg. 2012;36(4):793-799.

26. van Bockel JH, Geelkerken RH, Wasser MN. Chronic splanchnic ischaemia. Best Pract Res Clin Gastroenterol. 2001;15(1):99-119.

27. Geelkerken RH, van Bockel JH, de Roos WK, Hermans J, Terpstra JL. Chronic mesenteric vascular syndrome. Results of reconstructive surgery. Arch Surg. 1991;126(9):1101-1106.

28. Penna GL, Fialho FM, Kurtz P, et al. Changing sedative infusion from propofol to midazolam improves sublingual microcirculatory perfusion in patients with septic shock. J Crit Care. 2013;28(5):825-831.

29. Kanoore Edul VS, Ince C, Estenssoro E, et al. The Effects of Arterial Hypertension and Age on the Sublingual Microcirculation of Healthy Volunteers and Outpatients with Cardiovascular Risk Factors. Microcirculation. 2015;22(6):485-492.

30. Morozumi T, Kubota T, Sato T, Okuda K, Yoshie H. Smoking cessation increases gingival blood flow and gingival crevicular fluid. J Clin Periodontol. 2004;31(4):267-272.

31. Meekin TN, Wilson RF, Scott DA, Ide M, Palmer RM. Laser Doppler flowmeter measurement of relative gingival and forehead skin blood flow in light and heavy smokers during and after smoking. J Clin Periodontol. 2000;27(4):236-242.

32. Lindeboom JA, Mathura KR, Harkisoen S, van den Akker HP, Ince C. Effect of smoking on the gingival capillary density: assessment of gingival capillary density with orthogonal polarization spectral imaging. J Clin Periodontol. 2005;32(12):1208-1212.

33. Schraml C, Schwenzer NF, Martirosian P, Claussen CD, Schick F. Temporal course of perfusion in human masseter muscle during isometric contraction assessed by arterial spin labeling at 3T. MAGMA. 2011;24(4):201-209.

34. Harki J, Suker M, Duncel MS, et al. Sa2021 Patients With Chronic Gastrointestinal Ischemia Have an Altered Sublingual Microcirculation. Gastroenterology. 2016;150(4):S431-S432. 


\section{Supplementary materials}

Video SI Capturing sublingual microcirculation of a patient with chronic mesenteric ischemia before caloric challenge.

Video S2 Capturing sublingual microcirculation of a patient with chronic mesenteric ischemia after caloric challenge.

Video S3 Capturing the sublingual microcirculation of a healthy control before caloric challenge.

Video S4 Capturing the sublingual microcirculation of a healthy control after caloric challenge.

\section{Publish your work in this journal}

Clinical and Experimental Gastroenterology is an international, peerreviewed, open access, online journal publishing original research, reports, editorials, reviews and commentaries on all aspects of gastroenterology in the clinic and laboratory. This journal is included on PubMed. The manuscript management system is completely online

Submit your manuscript here: https://www.dovepress.com/clinical-and-experimental-gastroenterology-journal

and includes a very quick and fair peer-review system, which is all easy to use. Visit http://www.dovepress.com/testimonials.php to read real quotes from published authors. 